\title{
First report of Cryptosporidium spp. in white yaks in China
}

\author{
Si-Yuan Qin ${ }^{1,2 \dagger}$, Xiao-Xuan Zhang ${ }^{1,2+}$, Guang-Hui Zhao ${ }^{3}$, Dong-Hui Zhou ${ }^{1 *}$, Ming-Yang Yin ${ }^{1}$, Quan Zhao ${ }^{2}$
} and Xing-Quan Zhu ${ }^{1,2^{*}}$

\begin{abstract}
Background: Cryptosporidium is an enteric apicomplexan parasite, which can infect yaks, leading to reduction of milk production and poor weight gain. White yak (Bos grunniens) is a unique yak breed inhabiting only in Tianzhu Tibetan Autonomous County, Gansu province, northwestern China. The objective of the present study was to molecularly determine Cryptosporidium infection and species in white yaks.

Findings: Seventy-six fecal samples from white yaks in Tianzhu Tibetan Autonomous County, Gansu province were collected. The small subunit ribosomal RNA (SSU rRNA) gene of each sample was amplified using nested PCR and sequenced. The Cryptosporidium species was determined by comparison of the obtained sequences with that of corresponding Cryptosporidium sequences available in GenBank by BLAST (http://www.ncbi.nlm.nih.gov/BLAST/) and phylogenetic analysis with maximum likelihood (ML) using PAUP*. The overall prevalence of Cryptosporidium infection in white yak was 5.26\% (4/76). Species identification showed C. andersoni in one sample (collected in September), and C. bovis in three samples (one collected in November and two collected in September).

Conclusions: The present investigation revealed the existence of Cryptosporidium infection in white yaks in China, for the first time, and two Cryptosporidium species, namely C. andersoni and C. bovis, were identified. These findings extend the host range for Cryptosporidium spp., and also provide base-line information for further studies of molecular epidemiology and control of Cryptosporidium infection in the unique white yaks.
\end{abstract}

Keywords: Cryptosporidium spp, Genetic characterization, Prevalence, White Yak, China

\section{Findings \\ Background}

Cryptosporidium is an apicomplexan, enteric parasite pathogen, which may lead to diarrheal illness and other severe diseases of animals and humans [1,2]. Human infection with Cryptosporidium is usually through close contact with infected animals or consuming contaminated water or food [3]. Many animals can be infected with Cryptosporidium spp. including livestock and wild animals [4-8]. Four Cryptosporidium species have been identified in black yaks in previous studies [5,7].

\footnotetext{
*Correspondence: donghui822002@163.com; xingquanzhu1@hotmail.com †Equal contributors

'State Key Laboratory of Veterinary Etiological Biology, Key Laboratory of Veterinary Parasitology of Gansu Province, Lanzhou Veterinary Research Institute, Chinese Academy of Agricultural Sciences, Lanzhou, Gansu Province 730046, PR China

${ }^{2}$ College of Animal Science and Technology, Jilin Agricultural University, Changchun, Jilin Province 130118, PR China

Full list of author information is available at the end of the article
}

White yak (Bos grunniens) is a unique yak breed living only in Tianzhu Tibetan Autonomous County, Gansu province, northwestern China, where the air pressure is high and the temperature and oxygen content are low. White yak is known as the pearl of the plateau, and it is a rare and precious semi-wild animal of China and the world. Only approximately 49,400 white yaks were available in Tianzhu Tibetan Autonomous County [9]. Milk and meat of white yaks are the sought-after delicacy for local Tibetan people and other residents in Gansu Province. Previous studies have indicated that Cryptosporidium could infect black yaks, but it is yet to know whether white yaks are infected with Cryptosporidium spp.. The objective of the present study was to determine Cryptosporidium infection and species in white yaks in Tianzhu Tibetan Autonomous County, Gansu Province, China.

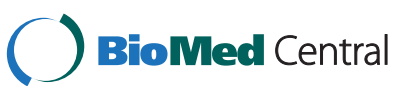

(C) 2014 Qin et al.; licensee BioMed Central Ltd. This is an Open Access article distributed under the terms of the Creative Commons Attribution License (http://creativecommons.org/licenses/by/4.0), which permits unrestricted use, distribution, and reproduction in any medium, provided the original work is properly credited. The Creative Commons Public Domain Dedication waiver (http://creativecommons.org/publicdomain/zero/1.0/) applies to the data made available in this article, unless otherwise stated. 


\section{Methods}

\section{Ethics statement}

This study was approved by the Animal Ethics Committee of Lanzhou Veterinary Research Institute, Chinese Academy of Agricultural Sciences (Approval No. LVRIAEC2013010). The white yaks from which the feces were collected, were handled in accordance with good animal practices required by the Animal Ethics Procedures and Guidelines of the People's Republic of China.

\section{The study site}

The fecal samples used in the present study were collected from white yaks in Tianzhu Tibetan Autonomous County, Gansu Province, northwestern China. The sampling site is between the eastern longitudes of $102^{\circ} 07^{\prime}$ $103^{\circ} 46^{\prime}$ and northern latitudes of $36^{\circ} 31^{\prime}-37^{\circ} 55^{\prime}$. The altitude of Tianzhu Tibetan Autonomous County ranges from $2,040 \mathrm{~m}$ to $4,878 \mathrm{~m}$, and the average annual temperature are from $-8^{\circ} \mathrm{C}$ to $4^{\circ} \mathrm{C}$, respectively.

\section{Specimen collection}

A total of 76 white yak fecal samples were randomly collected from two farms between September and November 2013 in Tianzhu Tibetan Autonomous County, Gansu Province, northwestern China. Information about the examined white yaks was obtained from the herdsmen at the time of sample collection. Fecal samples were collected with sterile gloves and transported to the laboratory in Lanzhou Veterinary Research Institute, Chinese Academy of Agricultural Sciences, Lanzhou, Gansu Province, China, and stored at $4^{\circ} \mathrm{C}$ within one week before being tested.

\section{DNA extraction and PCR amplification}

Genomic DNA was extracted from each fecal sample using the Stool DNA kit (OMEGA, USA) according to the manufacturer's instructions, and stored at $-20^{\circ} \mathrm{C}$ until PCR amplification. The small subunit ribosomal
RNA (SSU rRNA) gene was amplified using nested PCR to determine species/genotypes of Cryptosporidium spp. [4]. Positive and negative controls were included in each amplification. Amplification products were examined using 1.5\% agarose gel containing GoldView (Solarbio, China) and were observed under UV light.

\section{Sequencing and phylogenetic analyses}

All positive secondary PCR products were directly sequenced by Sangon Biotech Company (Shanghai, China). The obtained sequences were aligned with Cryptosporidium sequences available in GenBank database using the BLAST (http://www.ncbi.nlm.nih.gov/BLAST/) and Multiple Sequence Alignment Program, Clustal X 1.83 [4]. Phylogenetic relationships of Cryptosporidium spp. were reconstructed using Maximum Likelihood (ML) method, which were performed using a Fitch criterion within PAUP" (version 4.0b4a) and 1000 non-parametric bootstrap replicates. Plasmodium cathemerium (GenBank accession number: AY625607) was used as the out-group. The sequences obtained in the present study were deposited in GenBank with accession numbers of KJ094571 and KJ094572.

\section{Results and discussion}

Of 76 examined fecal samples of white yaks collected in Tianzhu Tibetan Autonomous County, Gansu province, 4 (5.26\%) were positive for Cryptosporidium infection. Sequencing and phylogenetic analysis identified two Cryptosporidium species present in these samples (Figure 1). One $C$. andersoni- and two C. bovis-positive samples were detected in September, and one C. bovis-positive sample was collected in November.

The overall Cryptosporidium prevalence in Tianzhu white yaks was 5.26\% (4/76) using a molecular approach, which was lower than that in yaks investigated by microscopy (10.4\%) [10], serological test (33.64\%) [11] and PCR

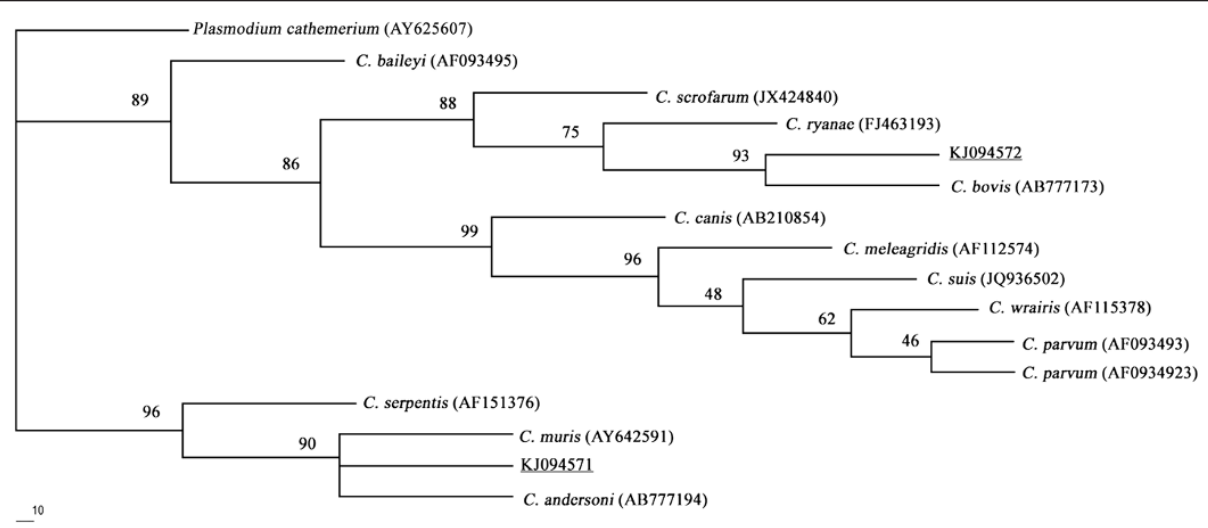

Figure 1 Phylogenetic analyses of Cryptosporidium spp. using Maximum Likelihood (ML) method based on sequences of the small subunit ribosomal RNA (SSU rRNA) gene. The numbers at clades indicate bootstrap values. The Cryptosporidium isolates identified in the present study are underlined. 
(24.2\%) [5] in Shanghai city and Qinghai province of China. The lower prevalence of Cryptosporidium infection in Tianzhu white yaks determined in the present investigation is likely due to the cold sampling seasons (September to November are becoming cold in Tianzhu Tibetan Autonomous County) and the small sample size.

Five Cryptosporidium species/genotypes, namely $C$. parvum [5], C. ryanae [5], Cryptosporidium sp. z13 [7], C. bovis [5] and deer-like genotype [12], have been detected in yaks in China. The present study revealed the presence of $C$. andersoni and C. bovis in white yaks. Of the four Cryptosporidium-positive samples, three represented $C$. bovis, indicating that $C$. bovis is the more prevalent Cryptosporidium species in white yaks, which is similar to previous studies that $C$. bovis is more prevalent in water buffaloes and beef calves [13,14].

However, C. parvum [5], C. ryanae [5], Cryptosporidium sp. z13 [7] and deer-like genotype [12] were not detected in white yaks in the present study, which may due to the small number of samples examined. C. parvum is one of the most important Cryptosporidium species, which has public health concerns [15], and it is a common species found in pre-weaned cattle in China and other countries [16-19]. Further studies will sample more white yaks in different seasons of the year to determine the dynamics and full profiles of Cryptosporidium infection in white yaks, to examine the infection status of the local Tibetans with Cryptosporidium, and to assess the zoonotic potential of Cryptosporidium from white yaks.

\section{Conclusions}

The present investigation revealed the existence of $C$. andersoni and C. bovis in Tianzhu white yaks, which is the first report of Cryptosporidium infection in Tianzhu white yaks. These results not only extend the host range for Cryptosporidium spp., but also provide base-line information for further studies of the molecular epidemiology and control of Cryptosporidium infection in Tianzhu white yaks.

\section{Competing interests}

The authors declare that they have no competing interests.

\section{Authors' contributions}

XQZ conceived and designed the study, and critically revised the manuscript. $S Y Q, X X Z, G H Z$ and $D H Z$ performed the experiments, analyzed the data and drafted the manuscript. MYY and QZ helped in study design, study implementation and manuscript revision. All authors read and approved the final manuscript.

\section{Acknowledgements}

This work was supported in part by the "Special Fund for Agro-scientific Research in the Public Interest" (Grant No. 201303037), the Science Fund for Creative Research Groups of Gansu Province (Grant No. 1210RJIA006), the Program for New Century Excellent Talents in University (Grant No. NCET-13-0489) and the Open Funds of the State Key Laboratory of Veterinary Etiological Biology, Lanzhou Veterinary Research Institute, Chinese Academy of Agricultural Sciences (Grant No. SKLVEB2013KFKT007).

\section{Author details}

'State Key Laboratory of Veterinary Etiological Biology, Key Laboratory of Veterinary Parasitology of Gansu Province, Lanzhou Veterinary Research Institute, Chinese Academy of Agricultural Sciences, Lanzhou, Gansu Province 730046, PR China. ${ }^{2}$ College of Animal Science and Technology, Jilin Agricultural University, Changchun, Jilin Province 130118, PR China. ${ }^{3}$ College of Veterinary Medicine, Northwest A\&F University, Yangling, Shaanxi Province, 712100, PR China.

Received: 29 March 2014 Accepted: 11 May 2014

Published: 19 May 2014

\section{References}

1. Cacciò SM, Pozio E: Advances in the epidemiology, diagnosis and treatment of cryptosporidiosis. Expert Rev Anti Infect Ther 2006, 4:429-443.

2. Xiao L: Overview of Cryptosporidium presentations at the 10th International Workshops on Opportunistic Protists. Eukaryot Cell 2009, 8:429-436.

3. Rossignol JF: Cryptosporidium and Giardia: treatment options and prospects for new drugs. Exp Parasitol 2010, 124:45-53.

4. Zhao GH, Ren WX, Gao M, Bian QQ, Hu B, Cong MM, Lin Q, Wang RJ, Qi M, Qi MZ, Zhu XQ, Zhang LX: Genotyping Cryptosporidium andersoni in cattle in Shaanxi Province, Northwestern China. PLoS One 2013, 8:e60112.

5. Mi R, Wang X, Li C, Huang Y, Zhou P, Li Z, Lei M, Cai J, Chen Z: Prevalence and genetic characterization of Cryptosporidium in yaks in Qinghai Province of China. PLoS One 2013, 8:e74985.

6. Yin J, Yuan Z, Shen Y, Zhang J, Jiang Y, Cao J: Molecular identification of Cryptosporidium spp. from animal sources in China. J Infect Dev Ctries 2013, 7:1020-1022.

7. Karanis P, Plutzer J, Halim NA, Igori K, Nagasawa H, Ongerth J, Liqing M: Molecular characterization of Cryptosporidium from animal sources in Qinghai province of China. Parasitol Res 2007, 101:1575-1580.

8. Wegayehu T, Adamu H, Petros B: Prevalence of Giardia duodenalis and Cryptosporidium species infections among children and cattle in North Shewa Zone, Ethiopia. BMC Infect Dis 2013, 13:419.

9. Ma C: Study on sustainable development strategies of Tianzhu white yak industry. Acta Ecol Anim Domast 2008, 29:133-135 (In Chinese).

10. Zhou C, He G, Zhang L: Investigation on Cryptosporidium infection in yaks. Chin J Zoonoses 2009, 25:389-390 (in Chinese).

11. Ma L, Lu Y, Cai Q, Wang G, Niu X, Ye X, Li X: Serological investigation of yak cryptosporidiosis in Qinghai Province. J Domest Anim Ecol 2011, 32:47-49 (in Chinese)

12. Feng $Y$, Ortega $Y$, He G, Das P, Xu M, Zhang X, Fayer R, Gatei W, Cama V, Xiao L: Wide geographic distribution of Cryptosporidium bovis and the deer-like genotype in bovines. Vet Parasitol 2007, 144:1-9.

13. Amer S, Zidan S, Feng Y, Adamu H, Li N, Xiao L: Identity and public health potential of Cryptosporidium spp. in water buffalo calves in Egypt. Vet Parasitol 2013, 191:123-127.

14. Nguyen ST, Fukuda Y, Tada C, Sato R, Duong B, Nguyen DT, Nakai Y: Molecular characterization of Cryptosporidium in native beef calves in central Vietnam. Parasitol Res 2012, 111:1817-1820.

15. Petry F, Jakobi V, Tessema TS: Host immune response to Cryptosporidium parvum infection. Exp Parasitol 2010, 126:304-309.

16. Zhang W, Wang R, Yang F, Zhang L, Cao J, Zhang X, Ling H, Liu A, Shen Y: Distribution and genetic characterizations of Cryptosporidium spp. in pre-weaned dairy calves in Northeastern China's Heilongjiang Province. PLoS One 2013, 8:e54857.

17. Murakoshi F, Xiao L, Matsubara R, Sato R, Kato Y, Sasaki T, Fukuda Y, Tada C, Nakai Y: Molecular characterization of Cryptosporidium spp. in grazing beef cattle in Japan. Vet Parasitol 2012, 187:123-128.

18. Xiao L: Molecular epidemiology of cryptosporidiosis: an update. Exp Parasitol 2010, 124:80-89.

19. Wang R, Wang H, Sun Y, Zhang L, Jian F, Qi M, Ning C, Xiao L: Characteristics of Cryptosporidium transmission in preweaned dairy cattle in Henan, China. J Clin Microbiol 2011, 49:1077-1082.

doi:10.1186/1756-3305-7-230

Cite this article as: Qin et al.: First report of Cryptosporidium spp. in white yaks in China. Parasites \& Vectors 2014 7:230. 